Research Article

\title{
Study on the Fracture Process Zone near the Mode I Dynamic Crack Tip
}

\author{
Chenmeng Ji (i) ${ }^{1}$ and Chengzhi Qi $\mathbb{i D}^{2}$ \\ ${ }^{1}$ College of Architectural and Civil Engineering, Beijing University of Technology, Beijing 100124, China \\ ${ }^{2}$ Beijing Advanced Innovation Center for Future Urban Design, \\ International Cooperation Base for Transportation Infrastructure Construction, \\ Beijing University of Civil Engineering and Architecture, Beijing 100044, China \\ Correspondence should be addressed to Chenmeng Ji; zuoshouti@sina.com
}

Received 19 January 2021; Revised 28 March 2021; Accepted 24 April 2021; Published 3 May 2021

Academic Editor: Francesco Aggogeri

Copyright (c) 2021 Chenmeng Ji and Chengzhi Qi. This is an open access article distributed under the Creative Commons Attribution License, which permits unrestricted use, distribution, and reproduction in any medium, provided the original work is properly cited.

\begin{abstract}
Evaluation of the shape and size of the fracture process zone near the mode I dynamic crack tip is still a problem unsolved completely at present. The research on the relationship between the fracture process zone and crack velocity near the mode I dynamic crack tip is quite limited, and some researchers have also developed experimental methods or numerical methods. In this research, based on the theory of elastodynamics and the complex stress function method, an approximate method for solving the mode I dynamic crack problem was proposed. The fracture process zone near the mode I dynamic crack tip was analyzed. The results showed that the areas of the fracture process zone determined based on the approximate method are nearly the same as the results obtained based on the well-known stress fields. The approximate method could provide a good reference for determining the fracture process zone near the mode I dynamic crack tip since no analytic methods had been found for evaluating the fracture process zone near the dynamic crack tip to the authors' knowledge.
\end{abstract}

\section{Introduction}

The study on the fracture process zone has been mainly focused on the fracture process zone near the static crack tip, while the analysis of the fracture process zone near the dynamic crack tip is very complicated due to the inertia and the dynamic effect of materials. For the fracture process zone near the static crack tip, some analytical models, such as the cohesion model [1], the virtual crack model [2], and twoparameter model [3], treat the fracture process zone as linear in form. The cell model proposed by Broberg [4-7] well explained the existence of the fracture process zone at the crack tip and provided a very visual explanation for future researchers. At present, the research studies on the dynamic crack tip fracture process zone are very limited, and some researchers have also developed experimental methods or numerical methods. For the determination of the fracture process zone near the dynamic crack tip, although Broberg
[8] and Fineberg [9] concluded that the fracture process zone at the dynamic crack tip increases with the increase of the crack velocity, the authors have searched relevant literature and found no relevant analytical methods yet. The determination of the profile of the fracture process zone at the dynamic crack tip is still an unsolved problem. The integral transformations with dual integral equation method and Wiener-Hopf method are difficult to allow for obtaining analytical solutions due to the complexity of calculations. The complex function method is much simpler than the integral transformation methods because it does not need to carry out the complex inversion operations.

In this study, based on the theory of elastodynamics and the complex stress function method, an approximate method for solving the dynamic crack problem was proposed. The fracture process zones at the mode I dynamic crack tip were analyzed. The contours and areas of the fracture process zones were determined by using the 
maximum tensile stress criterion for different crack velocities. Finally, the results based on the approximate method proposed in this paper were compared with the results calculated based on the well-known stress field at the crack tip to verify the feasibility and correctness of the approximate method.

\section{Method}

The crack tip is assumed to move along the direction of $x$ axis at a constant speed $V$. The far-field tension $\sigma$ is applied, see Figure 1. The fixed coordinate systems $\left(x_{1}, y, t\right)$ and the moving coordinate system $(x, y, t)$ have the following relationships:

$$
\left\{\begin{array}{l}
x=x_{1}-V t \\
y=y
\end{array}\right.
$$

The coordinate transformation gives

$$
\frac{\partial x}{\partial t}=-V \frac{\partial}{\partial x} .
$$

For plane elastic problem, the equation of motion takes the following form:

$$
\left\{\begin{array}{l}
\frac{\partial \sigma_{x}}{\partial x}+\frac{\partial \tau_{x y}}{\partial y}=\rho \frac{\partial^{2} u}{\partial t^{2}} \\
\frac{\partial \sigma_{y}}{\partial y}+\frac{\partial \tau_{x y}}{\partial x}=\rho \frac{\partial^{2} v}{\partial t^{2}}
\end{array}\right.
$$

where $\rho$ is mass density and $u$ and $v$ are displacements along $x$ and $y$ axes.

The relationships between stresses and strains give [10]

$$
\left\{\begin{array}{c}
\sigma_{x}=\lambda \theta+2 \mu \frac{\partial u}{\partial x} \\
\sigma_{y}=\lambda \theta+2 \mu \frac{\partial u}{\partial y}, \\
\tau_{x y}=\mu\left(\frac{\partial u}{\partial y}+\frac{\partial v}{\partial x}\right), \\
\theta=\frac{\partial u}{\partial x}+\frac{\partial v}{\partial y}
\end{array}\right.
$$

where $\mu$ and $\lambda$ are Lame's constants and $\theta$ is the first invariant of strain tensor, that is,

$$
\begin{aligned}
2 \mu \frac{\partial u}{\partial x} & =\sigma_{x}-\frac{\lambda}{2(\lambda+\mu)}\left(\sigma_{x}+\sigma_{y}\right), \\
2 \mu \frac{\partial v}{\partial y} & =\sigma_{y}-\frac{\lambda}{2(\lambda+\mu)}\left(\sigma_{x}+\sigma_{y}\right), \\
\theta & =\frac{1}{2(\lambda+\mu)}\left(\sigma_{x}+\sigma_{y}\right) .
\end{aligned}
$$

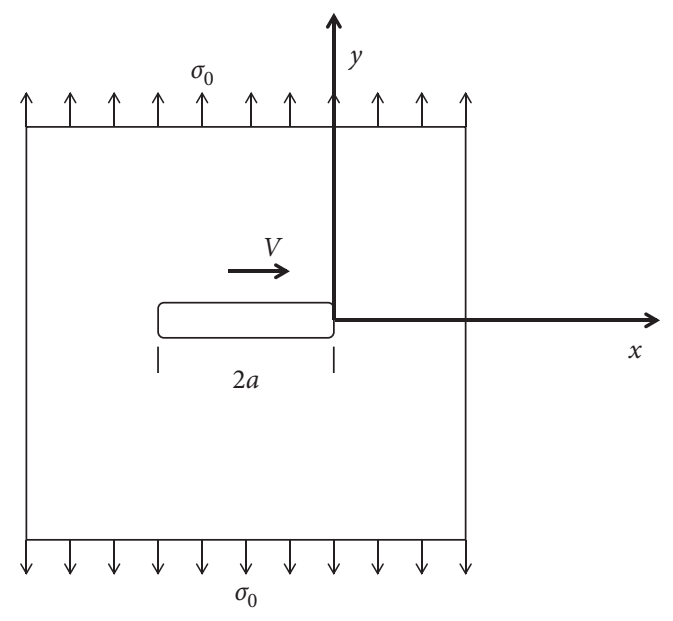

Figure 1: Model of mode I crack. [10]

The equation of compatibility takes the form as follows

$$
\left(\frac{\partial^{2}}{\partial x^{2}}+\frac{\partial^{2}}{\partial y^{2}}\right)\left(\sigma_{x}+\sigma_{y}\right)-\frac{2(\lambda+\mu)}{\lambda+2 \mu} \rho \frac{\partial^{2}}{\partial t^{2}}\left(\frac{\partial u}{\partial x}+\frac{\partial v}{\partial y}\right)=0 .
$$

Substituting equation (5) to equation (6), the following equation can be obtained:

$$
\left(\frac{\partial^{2}}{\partial x^{2}}+\frac{\partial^{2}}{\partial y^{2}}-\frac{\rho}{\lambda+2 \mu} \frac{\partial^{2}}{\partial t^{2}}\right)\left(\sigma_{x}+\sigma_{y}\right)=0
$$

Taking partial derivatives with respect to $x$ and $y$ for both sides of equation (3), the following equations can be obtained:

$$
\begin{aligned}
& \left\{\begin{array}{l}
\frac{\partial^{2} \sigma_{x}}{\partial x \partial y}+\frac{\partial^{2} \tau_{x y}}{\partial y^{2}}=\rho \frac{\partial^{3} u}{\partial t^{2} \partial y} \\
\frac{\partial^{2} \sigma_{y}}{\partial x \partial y}+\frac{\partial^{2} \tau_{x y}}{\partial x^{2}}=\rho \frac{\partial^{2} v}{\partial t^{2} \partial x}
\end{array}\right. \\
& \left\{\begin{array}{l}
\frac{\partial^{2} \sigma_{x}}{\partial x^{2}}+\frac{\partial^{2} \tau_{x y}}{\partial x \partial y}=\rho \frac{\partial^{3} u}{\partial t^{2} \partial x} \\
\frac{\partial^{2} \sigma_{y}}{\partial y^{2}}+\frac{\partial^{2} \tau_{x y}}{\partial x \partial y}=\rho \frac{\partial^{3} v}{\partial t^{2} \partial y}
\end{array}\right.
\end{aligned}
$$

By adding and subtracting both sides of the above two groups of equations, the following can be obtained:

$$
\frac{\partial^{2} \sigma_{x}}{\partial x^{2}}-\frac{\partial^{2} \sigma_{y}}{\partial y^{2}}=\frac{\rho}{2 \mu} \frac{\partial^{2}}{\partial t^{2}}\left(\sigma_{x}-\sigma_{y}\right) \text {. }
$$

Rearranging equation (9) yields

$$
\left(\frac{\partial^{2}}{\partial x^{2}}-\frac{\rho}{2 \mu} \frac{\partial^{2}}{\partial t^{2}}\right) \sigma_{x}=\left(\frac{\partial^{2}}{\partial y^{2}}-\frac{\rho}{2 \mu} \frac{\partial^{2}}{\partial t^{2}}\right) \sigma_{y} .
$$


Obviously, introducing the stress function $U$ yields the following equations [10]:

$$
\begin{aligned}
\sigma_{x} & =\left(\frac{\partial^{2}}{\partial y^{2}}-\frac{\rho}{2 \mu} \frac{\partial^{2}}{\partial t^{2}}\right) U, \\
\sigma_{y} & =\left(\frac{\partial^{2}}{\partial x^{2}}-\frac{\rho}{2 \mu} \frac{\partial^{2}}{\partial t^{2}}\right) U .
\end{aligned}
$$

Adding the above two equations yields the following equation:

$$
\sigma_{x}+\sigma_{y}=\left(\frac{\partial^{2}}{\partial y^{2}}+\frac{\partial^{2}}{\partial x^{2}}-\frac{\rho}{\mu} \frac{\partial^{2}}{\partial t^{2}}\right) U
$$

Substituting equation (12) into equation (7) yields

$$
\left(\frac{\partial^{2}}{\partial x^{2}}+\frac{\partial^{2}}{\partial y^{2}}-\frac{\rho}{\lambda+2 \mu} \frac{\partial^{2}}{\partial t^{2}}\right)\left(\frac{\partial^{2}}{\partial x^{2}}+\frac{\partial^{2}}{\partial y^{2}}-\frac{\rho}{\mu} \frac{\partial^{2}}{\partial t^{2}}\right) U=0 .
$$

By substituting some variables, equation (13) is transformed as follows:

$$
\left(\frac{\partial^{2}}{\partial x^{2}}+\frac{\partial^{2}}{\partial y^{2}}-\frac{1}{c_{1}^{2}} \frac{\partial^{2}}{\partial t^{2}}\right)\left(\frac{\partial^{2}}{\partial x^{2}}+\frac{\partial^{2}}{\partial y^{2}}-\frac{1}{c_{2}^{2}} \frac{\partial^{2}}{\partial t^{2}}\right) U=0,
$$

where $c_{1}$ is the longitudinal wave velocity and $c_{2}$ is transverse wave velocity, which are defined as follows:

$$
\left\{\begin{array}{l}
c_{1}=\sqrt{\frac{\lambda+2 \mu}{\rho}}, \\
c_{2}=\sqrt{\frac{\mu}{\rho}}
\end{array}\right.
$$

According to equations (2) and (14), it can be changed to the form as follows:

$$
\left(\frac{\partial^{2}}{\partial x^{2}}+\frac{\partial^{2}}{\partial y_{1}^{2}}\right)\left(\frac{\partial^{2}}{\partial x^{2}}+\frac{\partial^{2}}{\partial y_{2}^{2}}\right) U=0,
$$

where

$$
\begin{aligned}
& \left\{\begin{array}{l}
\alpha_{1}=\sqrt{1-\frac{V^{2}}{c_{1}^{2}}}, \\
\alpha_{2}=\sqrt{1-\frac{V^{2}}{c_{2}^{2}}},
\end{array}\right. \\
& \left\{\begin{array}{l}
y_{1}=\alpha_{1} y, \\
y_{2}=\alpha_{2} y .
\end{array}\right.
\end{aligned}
$$

Since the analytical solution of equation (16) cannot be obtained accurately, and the expression of the stress function of the dynamic crack cannot be determined, the stress field near the mode I crack tip cannot be determined directly by solving equation (16). The controlling equation of the static problem is one biharmonic equation, and the stress function method for solving static fracture process zone near the crack tip is already very complicated. Equation (16) is very similar in form to the biharmonic equation. Hence, we consider using the method for solving static crack problems to solve the dynamic problem of the stress field near the crack tip.

Let

$$
\bar{y}=\sqrt{\frac{2}{\left(1 / \alpha_{1}^{2}\right)+\left(1 / \alpha_{2}^{2}\right)}} y=\sqrt{\frac{2 \alpha_{1}^{2} \alpha_{2}^{2}}{\alpha_{1}^{2}+\alpha_{2}^{2}} y .}
$$

So, equation (16) can be rearranged as follows:

$$
\left(\frac{\partial^{4}}{\partial x^{4}}+2 \frac{\partial^{4}}{\partial x^{2} \bar{y}^{2}}+\frac{\partial^{4}}{\partial \bar{y}^{4}}\right) U+\left[\frac{4 \alpha_{1}^{2} \alpha_{2}^{2}}{\left(\alpha_{1}^{2}+\alpha_{2}^{2}\right)^{2}}-1\right] \frac{\partial^{4}}{\partial \bar{y}^{4}} U=0 .
$$

Let

$$
\beta=\frac{4 \alpha_{1}^{2} \alpha_{2}^{2}}{\left(\alpha_{1}^{2}+\alpha_{2}^{2}\right)^{2}}-1
$$

So, equation (19) can be rearranged as follows:

$$
\left(\frac{\partial^{2}}{\partial x^{2}}+\frac{\partial^{2}}{\partial \bar{y}^{2}}\right)^{2} U+\beta \frac{\partial^{4}}{\partial \bar{y}^{4}} U=0
$$

It can be seen that equation (21) is a fourth-order partial differential equation about $x$ and $\bar{y}$. It is very difficult to solve equation (21) without some efforts. However, the second term on the left side of the equation contains parameter $\beta$, and the values of $\beta$ at different crack velocity are shown in Table 1.

It can be seen from Table 1 that $\beta$ increases with the crack velocity. The maximum value of $\beta$ is reached when the Rayleigh wave velocity is approached. However, the generally observed maximum crack propagation velocity in experiments is $0.34-0.45$ times of the Rayleigh wave velocity, and the value of $\beta$ is in the range of -0.00025 to -0.00139 . The value of $\beta$ is very small, and the second term of equation (21) can be neglected. Hence, the following equation can be used to explore the approximate solution of equation (16):

$$
\left(\frac{\partial^{2}}{\partial x^{2}}+\frac{\partial^{2}}{\partial \bar{y}^{2}}\right)^{2} U=0
$$

At this time, equation (15) is the biharmonic equation in the coordinate system $(x \bar{y})$ and has the same form as that for the static crack. Then, we can find the approximate solution for the dynamic crack by using the stress function method.

For mode I crack, Westergaard [11] assumed the stress function taking the following form:

$$
U(x, y)=\operatorname{Re}\left(\int \mathrm{d} z \int Z(z) \mathrm{d} z\right)+y \operatorname{Im}\left(\int Z(z) \mathrm{d} z\right) .
$$

The complex stress function is $[12,13]$ 
TABLe 1: The values of $\beta$ for different velocities.

\begin{tabular}{lccccc}
\hline$V / c$ & $\alpha_{1}$ & $\alpha_{2}$ & $\alpha_{1}^{2}$ & $\alpha_{2}^{2}$ & $\beta$ \\
\hline 0 & 1 & 1 & 1 & 1 & 0 \\
0.19 & 0.9945 & 0.9984 & 0.9891 & 0.9968 & $-1.53 E-05$ \\
0.38 & 0.9780 & 0.9937 & 0.9565 & 0.9875 & -0.00025 \\
0.57 & 0.9498 & 0.9859 & 0.9021 & 0.972 & -0.00139 \\
0.76 & 0.9088 & 0.9748 & 0.8260 & 0.9503 & -0.00490 \\
0.95 & 0.8533 & 0.9604 & 0.7281 & 0.9224 & -0.01386 \\
\hline
\end{tabular}

$$
Z(z)=\frac{K_{1}}{\sqrt{2 \pi z_{1}}}\left[1+\frac{3}{4}\left(\frac{z}{a}\right)-\frac{5}{32}\left(\frac{z}{a}\right)^{2}+\frac{21}{384}\left(\frac{z}{a}\right)^{3}-\ldots\right]
$$

Then, the stress fields near the mode I crack tip can be calculated:

$$
\left\{\begin{aligned}
\sigma_{x} & =\operatorname{Re} Z-\bar{y} \operatorname{Im} Z^{\prime}, \\
\sigma_{y} & =\operatorname{Re} Z+\bar{y} \operatorname{Im} Z^{\prime}, \\
\tau_{x y} & =-\bar{y} \operatorname{Re} Z^{\prime} .
\end{aligned}\right.
$$

The size of the FPZ can be calculated theoretically based on the appropriate criterion and the crack tip stress field. The maximum tensile stress criterion for brittle materials can be expressed as follows:

$$
\sigma_{1}=\frac{\sigma_{x}+\sigma_{y}}{2}+\sqrt{\left(\frac{\sigma_{x}-\sigma_{y}}{2}\right)^{2}+\tau_{x y}^{2}}=\sigma_{u},
$$

where $\sigma_{u}$ is the tensile strength and is assumed to be independent of the crack velocity.

When more than one term in the expansion of the stress function is taken, the expressions for the stress field based on the stress function method at the crack tip are shown in Appendix.

\section{Results}

By substituting equation (25) into equation (26), the implicit equation of the FPZ can be easily obtained. As an example, we take the following material parameters: the longitudinal wave velocity $c_{1}=5370 \mathrm{~m} / \mathrm{s}$, the shear wave velocity $c_{2}=3180 \mathrm{~m} / \mathrm{s}$, and the Rayleigh wave velocity $c=2900 \mathrm{~m} / \mathrm{s}$. The contours of the FPZ constructed by taking different terms of the stress function of equation (24) for different crack velocities are shown in Figure 2.

From Figure 2, it can be seen that the fracture process zone increases with the increasing crack velocity. The higher the velocity is, the more significant the change of the fracture process zone is. The area of the fracture process zone at $0.38 c$ is 1.026 times that at $0.19 c$, and the area of the fracture process zone at $0.76 c$ is 1.34 times that at $0.95 c$, or 1.657 times that at $0.19 c$. Taking the velocity of $0.38 c$ as an example, the calculated areas of the fracture process zone are shown in Table 2. The area of the fracture process zone calculated by the first two terms of the stress function is the largest. The more the stress function terms are taken, the smaller the change rate of the area of the fracture process zone at the crack tip is, which also indicates that the calculation results become more and more stable.

\section{Discussion}

The approximate method is proposed in the second part by ignoring the influence of the less-significant parameters. The well-known stress field was used to calculate the fracture process zone at the crack tip, and the results by using the approximate method were compared to the results by using the well-known stress fields to verify the correctness of the approximate method. The expressions for the mode I stress fields are given as follows [14]:

$$
\left\{\begin{array}{l}
\sigma_{x}=\sum \frac{K_{n}^{0} B_{I}(V)}{\sqrt{2 \pi}} \frac{n(n+1)}{2}\left[\left(1+2 \alpha_{1}^{2}-\alpha_{2}^{2}\right) r_{1}^{(n / 2)-1} \cos \frac{(n-1) \theta_{1}}{2}-2 h(n) r_{2}^{(n / 2)-1} \cos \frac{(n-1) \theta_{2}}{2}\right], \\
\sigma_{y}=\sum \frac{K_{n}^{0} B_{I}(V)}{\sqrt{2 \pi}} \frac{n(n+1)}{2}\left[-\left(1+\alpha_{2}^{2}\right) r_{1}^{(n / 2)-1} \cos \frac{(n-1) \theta_{1}}{2}+2 h(n) r_{2}^{(n / 2)-1} \cos \frac{(n-1) \theta_{2}}{2}\right], \\
\tau_{x y}=\sum \frac{K_{n}^{0} B_{I}(V)}{\sqrt{2 \pi}} \frac{n(n+1)}{2}\left[-2 \alpha_{1} r_{1}^{(n / 2)-1} \sin \frac{(n-1) \theta_{1}}{2}+\frac{1+\alpha_{2}^{2}}{\alpha_{2}} h(n) r_{2}^{(n / 2)-1} \sin \frac{(n-1) \theta_{2}}{2}\right],
\end{array}\right.
$$

where 


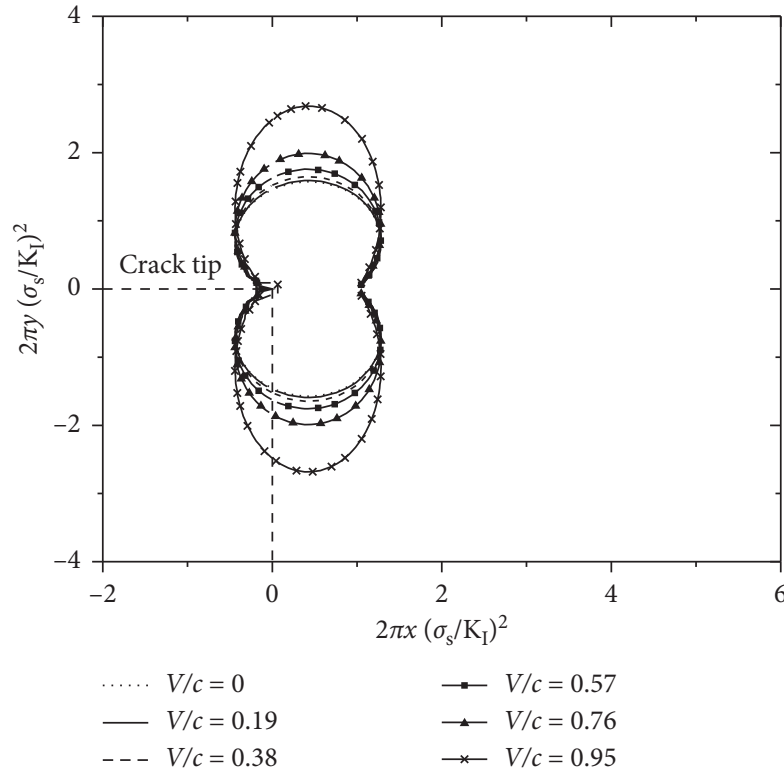

(a)

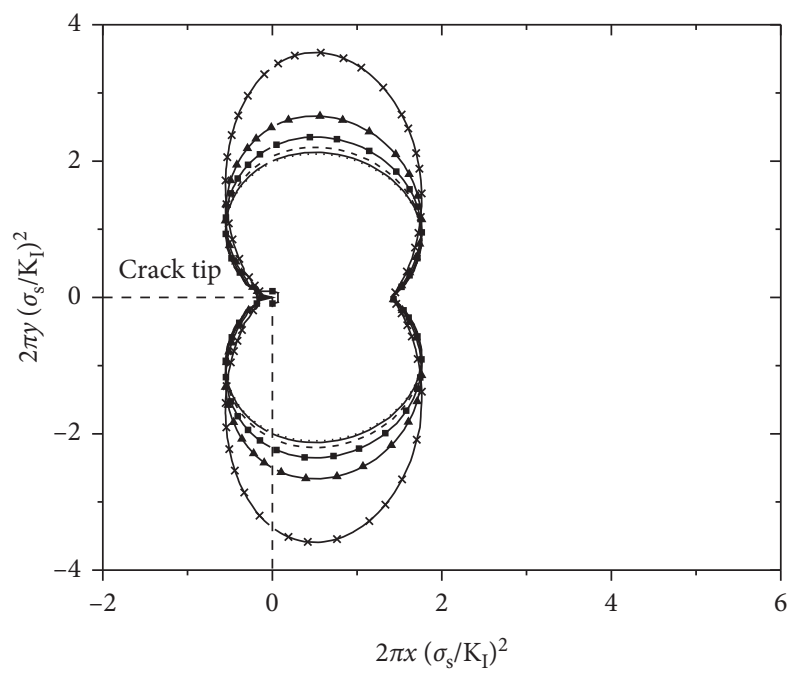

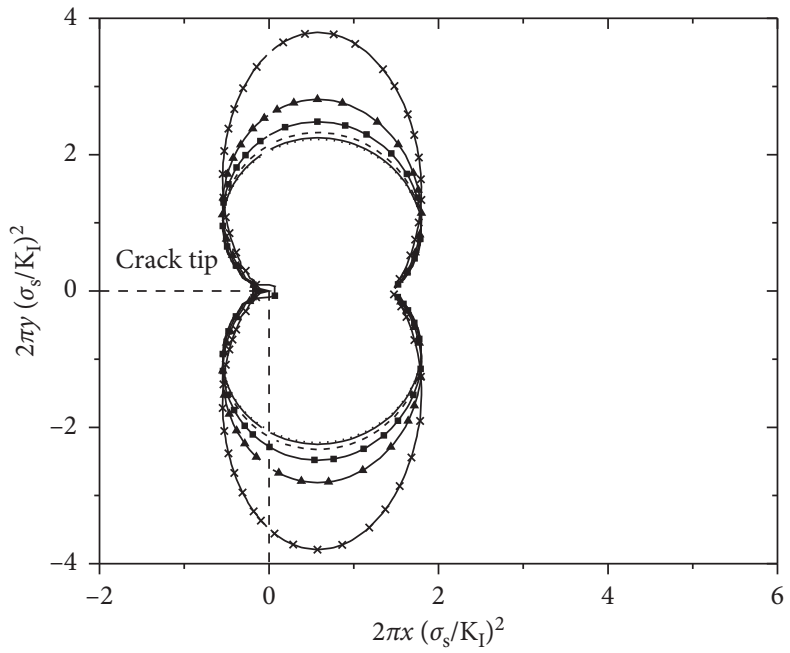

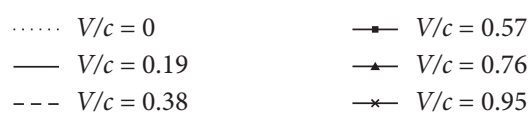

(b)

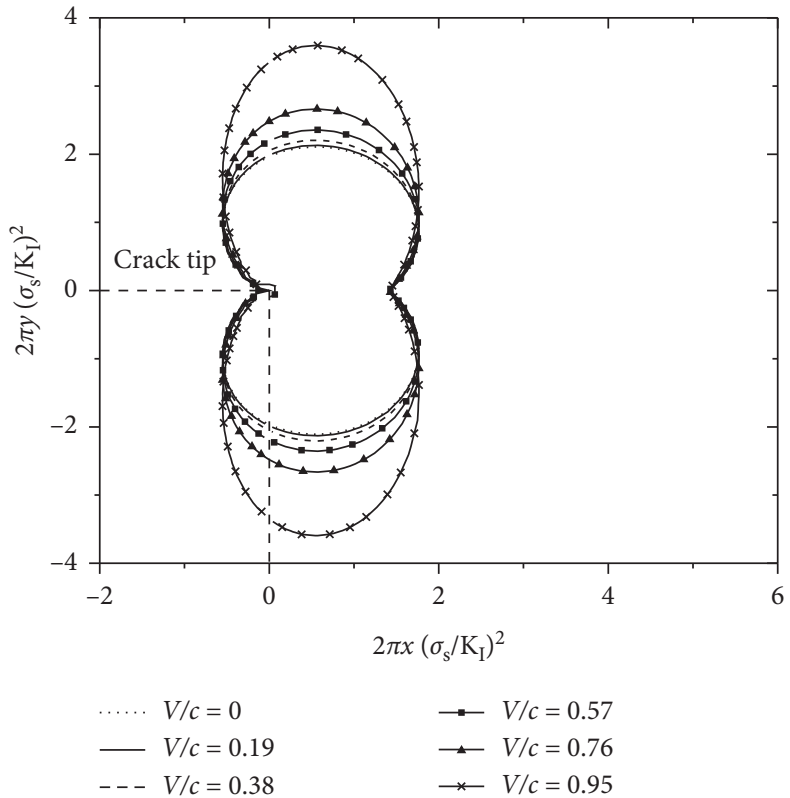

(d)

FIGURE 2: The FPZs constructed by using the maximum tensile stress criterion with different terms of stress function: (a) only first term, (b) first two terms, (c) first three terms, and (d) first four terms.

TABLE 2: Areas of the FPZ for different terms in the expansion of the stress function.

\begin{tabular}{lcc}
\hline Stress function & Area of the fracture process zone $\left(\mathrm{cm}^{2}\right)$ & Change rate $(\%)$ \\
\hline First term & 4.23987 & - \\
First two terms & 7.81604 & 84.35 \\
First three terms & 7.31473 & 6.41 \\
First four terms & 7.32698 & 0.167 \\
First five terms & 7.32314 & 0.052 \\
\hline
\end{tabular}


TABLE 3: Comparison of the FPZ areas by using the approximate method and the method based on the well-known stress $\left(\mathrm{cm}^{2}\right)$.

\begin{tabular}{lccccccc}
\hline & Crack velocity (m/s) & 100 & 300 & 500 & 700 & 900 & 1100 \\
\hline \multirow{2}{*}{ First term } & Well-known stress method & 4.0947 & 4.1645 & 4.2669 & 4.4268 & 4.6940 & 5.0909 \\
& Approximate method & 3.9780 & 4.0031 & 4.0578 & 4.0930 & 4.1400 & 4.2102 \\
\hline \multirow{2}{*}{ First two terms } & Well-known stress method & 6.2264 & 6.3738 & 6.6077 & 7.0034 & 7.6029 & 8.5297 \\
& Approximate method & 7.4694 & 7.4774 & 7.5042 & 7.5712 & 7.6678 & 7.7990 \\
\hline
\end{tabular}

$$
\begin{aligned}
& B_{I}(V)=\frac{1+\alpha_{2}^{2}}{4 \alpha_{1} \alpha_{2}-\left(1+\alpha_{2}^{2}\right)^{2}}, \\
& h(n)= \begin{cases}\frac{2 \alpha_{1} \alpha_{2}}{1+\alpha_{2}^{2}}, & \text { odd, } \\
\frac{1+\alpha_{2}^{2}}{2}, & \text { even, }\end{cases} \\
& \bar{x}=x_{1}-\dot{a} t=\bar{r}_{1} \cos \theta_{1}=\bar{r}_{2} \cos \theta_{2}, \\
& \bar{y}=y=\frac{r_{1}}{a} \sin \theta_{1}=\frac{r_{2}}{a} \sin \theta_{2}, \\
& r_{1}=\sqrt{\bar{x}^{2}+\alpha_{1}^{2} \bar{y}^{2}}, r_{2}=\sqrt{\bar{x}^{2}+\alpha_{2}^{2} \bar{y}^{2}}, \\
& \theta_{1}=\arctan \left(\alpha_{1} \frac{\bar{y}}{\bar{x}}\right), \theta_{2}=\arctan \left(\alpha_{2} \frac{\bar{y}}{\bar{x}}\right)
\end{aligned}
$$

If equation (27) is substituted into equation (26), we can obtain the equation for the fracture process zone at the crack tip $r=f(\theta)$. The higher-order terms of the stress field have little effect on the stress field near the crack tip of mode I, but the second term has a significant effect on the fracture process zone at the crack tip. Therefore, the first term and the first two terms in the stress field expansion expression are considered, respectively, to calculate the fracture process zone at the crack tip of mode I. $K_{2}{ }^{0}$ is a parameter similar to the stress intensity factor, which is difficult to determine. Because the dependence of T-stress on the velocity for dynamic crack is not clear, the ratio of T-stress to the far-field stress is 0.526 referred to the work by Seed and Nowell [15].

When the first term and the first two terms of the stress function expansion are taken respectively, the comparisons of the areas of the fracture process zone at the mode I crack tip calculated based on the approximate method and the well-known stress field expression are shown in Table 3. It can be seen that the results of the mode I crack tip fracture process zone calculated by the approximate method are highly consistent with those of the crack tip fracture process zone calculated based on the known stress field, and both increase with the increase of the crack velocity. When the velocity is relatively high, the area of the fracture process zone at the mode I crack tip calculated by the approximate method is slightly smaller than that calculated based on the well-known stress field, which is believed to be caused by neglecting the influence of small parameters $\beta$. The comparison of the calculation results of the two methods in Table 3 verifies the correctness of the approximate method in this paper for evaluating the fracture process zone at the tip of mode I dynamic crack, which can provide a reference for the study of the mode I dynamic crack problem.

\section{Conclusions}

An approximate method was proposed by transforming the governing equation and disregarding the less-significant parameter. The areas and sizes of the fracture process zone of the mode I dynamic crack were evaluated by the approximate method. The correctness of the method was verified by comparing the results calculated by the approximate method and the well-known stress fields. The conclusions can be drawn as follows:

(1) The fracture process zone near the mode I dynamic crack tip distributed symmetrically about the crack surface. The higher the crack propagation velocity, the larger the size of the FPZ for the mode I crack. When the velocity approached the Rayleigh wave velocity, the fracture process zone changed more significantly.

(2) The fracture process zone can be calculated by using this approximate method with different terms of the stress function. The sizes and areas of the fracture process zone calculated by the proposed method were very close to those calculated based on the wellknown stress field. Therefore, the proposed method is suitable for the mode I dynamic crack.

(3) Although the well-known stress field is widely accepted, it is just an asymptotic solution for the mode I dynamic crack tip, i.e., $n=1$. When $n>1$, the parameter $K_{2}{ }^{0}$ cannot be determined except by experiment. This means that it is difficult to use the well-known stress field expression to evaluate the FPZ for the dynamic crack tip for $n>1$. However, the approximate method can be used to obtain the results with different accuracies by taking different terms from the expansion of the stress function. It is also noteworthy that the method is very easy to implement for determining the shape and size of the FPZ of the mode I dynamic crack.

\section{Appendix}

In this appendix, the authors will show the expression of the stress field used in the calculations. 
Let

$$
\begin{aligned}
& M=\frac{\sqrt{\left(2 \alpha_{1}^{2} \alpha_{2}^{2} / \alpha_{1}^{2}+\alpha_{2}^{2}\right)} y}{x}, \\
& P=\frac{2 \alpha_{1}^{2} \alpha_{2}^{2} y^{2}}{\alpha_{1}^{2}+\alpha_{2}^{2}}+x^{2}, \\
& Q=\sqrt{\frac{\alpha_{1}^{2} \alpha_{2}^{2}}{\alpha_{1}^{2}+\alpha_{2}^{2}}}
\end{aligned}
$$

$$
\begin{aligned}
\sigma_{x} & =\frac{-y \sin \left((3 / 2) \tan ^{-1} M\right) K_{1} Q}{2 \sqrt{\pi} P^{(3 / 3)}}+\frac{\cos \left((1 / 2) \tan ^{-1} M\right) K_{1}}{\sqrt{2 \pi} P^{(1 / 4)}}, \\
\sigma_{y} & =\frac{y \sin \left((3 / 2) \tan ^{-1} M\right) K_{1} Q}{2 \sqrt{\pi} P^{(3 / 4)}}+\frac{\cos \left((1 / 2) \tan ^{-1} M\right) K_{1}}{\sqrt{2 \pi} P^{(1 / 4)}}, \\
\sigma_{x y} & =\frac{y \cos \left((3 / 2) \tan ^{-1} M\right) K_{1} Q}{2 \sqrt{\pi} P^{(3 / 4)}} .
\end{aligned}
$$

So, the stress fields for taking the first term of the ex-

When the first two terms of the expansions of the stress functions are taken, the stress fields near mode are

$$
\begin{aligned}
\sigma_{x}= & \frac{-y K_{1} Q\left[\sqrt{2}(4 a-3 x) \sin \left((3 / 2) \tan ^{-1} M\right)+6 y \cos \left((3 / 2) \tan ^{-1} M\right) Q\right]}{8 a \sqrt{2 \pi} P^{(3 / 4)}} \\
& +\frac{K_{1}\left[\sqrt{2}(4 a+3 x) \cos \left((1 / 2) \tan ^{-1} M\right)+6 y \sin \left((1 / 2) \tan ^{-1} M\right) Q\right]}{8 a \sqrt{\pi} P^{(1 / 4)}}, \\
\sigma_{y}= & \frac{y K_{1} Q\left[\sqrt{2}(4 a-3 x) \sin \left((1 / 2) \tan ^{-1} M\right)+6 y \cos \left((3 / 2) \tan ^{-1} M\right) Q\right]}{8 a \sqrt{2 \pi} P^{(3 / 4)}} \\
& +\frac{K_{1}\left[\sqrt{2}(4 a+3 x) \cos \left((1 / 2) \tan ^{-1} M\right)+6 y \sin \left((1 / 2) \tan ^{-1} M\right) Q\right]}{8 a \sqrt{\pi} P^{(1 / 4)}}, \\
\sigma_{x y}= & \frac{-y K_{1} Q\left[-\sqrt{2}(4 a-3 x) \cos \left((3 / 2) \tan ^{-1} M\right)+6 y \sin \left((3 / 2) \tan ^{-1} M\right) Q\right]}{8 a \sqrt{2 \pi} P^{(3 / 4)}} .
\end{aligned}
$$

The expressions of the stress fields for the mode crack when taking first three terms are shown as follows:

$$
\begin{gathered}
\sigma_{x}=\frac{-y K_{1} Q\left[\sqrt{2} \alpha_{1}^{2}\left(32 a^{2}-24 a x+15 x^{2}-30 \alpha_{2}^{2} y^{2}\right) \sin \left((3 / 2) \tan ^{-1} M\right)+\sqrt{2} \alpha_{2}^{2}\left(32 a^{2}-24 a x+15 x^{2}\right) \sin \left((3 / 2) \tan ^{-1} M\right)+12 Q y(4 a-5 x) \cos \left((3 / 2) \tan ^{-1} M\right)\right]}{64 \sqrt{\pi} a^{2}\left(\alpha_{1}^{2}+\alpha_{2}^{2}\right) P^{(3 / 4)}}+ \\
\frac{K_{1}\left[\sqrt{2} \alpha_{1}^{2}\left(32 a^{2}+24 a x-15 x^{2}+10 \alpha_{2}^{2} y^{2}\right) \cos \left((1 / 2) \tan ^{-1} M\right)+\sqrt{2} \alpha_{2}^{2}\left(32 a^{2}+24 a x-5 x^{2}\right) \cos \left((1 / 2) \tan ^{-1} M\right)+4 Q y(12 a-5 x) \sin \left((1 / 2) \tan ^{-1} M\right)\right]}{64 \sqrt{2 \pi} a^{2}\left(\alpha_{1}^{2}+\alpha_{2}^{2}\right) P^{(1 / 4)}}, \\
\sigma_{y}=\frac{y K_{1} Q\left[\sqrt{2} \alpha_{1}^{2}\left(32 a^{2}-24 a x+15 x^{2}-30 \alpha_{2}^{2} y^{2}\right) \sin \left((3 / 2) \tan ^{-1} M\right)+\sqrt{2} \alpha_{2}^{2}\left(32 a^{2}-24 a x+15 x^{2}\right) \sin _{(}\left((3 / 2) \tan ^{-1} M\right)+12 Q y(4 a-5 x) \cos \left((3 / 2) \tan ^{-1} M\right)\right]}{64 \sqrt{\pi} a^{2}\left(\alpha_{1}^{2}+\alpha_{2}^{2}\right) P^{(3 / 4)}}+ \\
\frac{K_{1}\left[\sqrt{2} \alpha_{1}^{2}\left(32 a^{2}+24 a x-15 x^{2}+10 \alpha_{2}^{2} y^{2}\right) \cos \left((1 / 2) \tan ^{-1} M\right)+\sqrt{2} \alpha_{2}^{2}\left(32 a^{2}+24 a x-5 x^{2}\right) \cos \left((1 / 2) \tan ^{-1} M\right)+4 Q y(12 a-5 x) \sin \left((1 / 2) \tan ^{-1} M\right)\right]}{64 \sqrt{2 \pi} a^{2}\left(\alpha_{1}^{2}+\alpha_{2}^{2}\right) P^{(1 / 4)}}, \\
\sigma_{x y}=\frac{-y K_{1} Q\left[-\sqrt{2} \alpha_{1}^{2}\left(32 a^{2}-24 a x+15 x^{2}-30 \alpha_{2}^{2} y^{2}\right) \cos \left((3 / 2) \tan ^{-1} M\right)-\sqrt{2} \alpha_{2}^{2}\left(32 a^{2}-24 a x+15 x^{2}\right) \cos \left((3 / 2) \tan ^{-1} M\right)+12 Q y(4 a-5 x) \sin _{(}\left((3 / 2) \tan ^{-1} M\right)\right]}{64 \sqrt{\pi} a^{2}\left(\alpha_{1}^{2}+\alpha_{2}^{2}\right) P^{(3 / 4)}}+
\end{gathered}
$$

The stress fields for the first four terms of the stress function are shown as follows: 


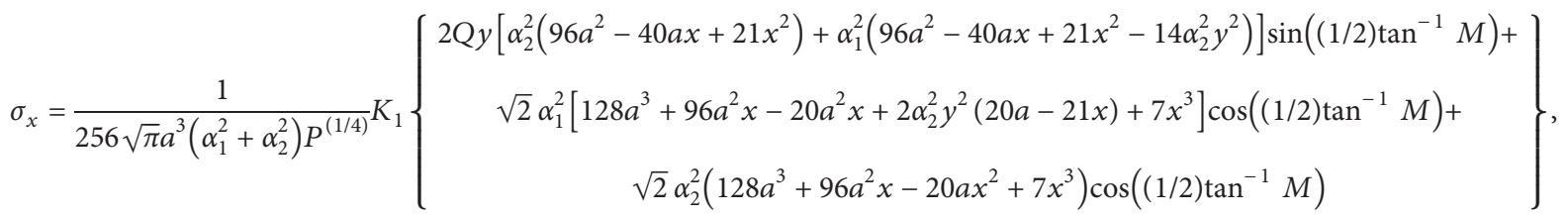

$$
\begin{aligned}
& -\frac{1}{256 \sqrt{\pi} a^{3}\left(\alpha_{1}^{2}+\alpha_{2}^{2}\right) P^{(3 / 4)}} Q y K_{1}\left\{\begin{array}{c}
2 Q y\left[3 \alpha_{2}^{2}\left(32 a^{2}-40 a x+35 x^{2}\right)+\alpha_{1}^{2}\left(96 a^{2}-120 a x+105 x^{2}-70 \alpha_{2}^{2} y^{2}\right)\right] \cos \left((3 / 2) \tan ^{-1} M\right)+ \\
\sqrt{2} \alpha_{1}^{2}\left[128 a^{3}-96 a^{2} x+60 a^{2} x-30 \alpha_{2}^{2} y^{2}(4 a-7 x)-35 x^{3}\right] \sin \left((3 / 2) \tan ^{-1} M\right)+ \\
\sqrt{2} \alpha_{2}^{2}\left(128 a^{3}-96 a^{2} x+60 a x^{2}-35 x^{3}\right) \sin \left((3 / 2) \tan ^{-1} M\right)
\end{array}\right\},
\end{aligned}
$$

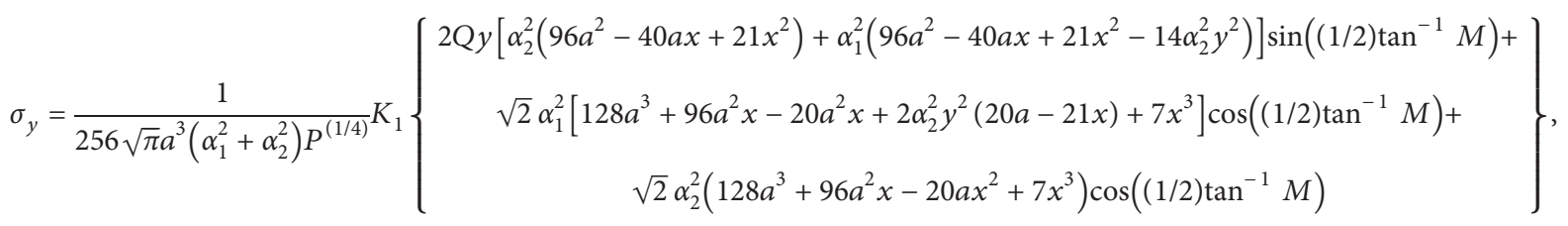

$$
\begin{aligned}
& +\frac{1}{256 \sqrt{\pi} a^{3}\left(\alpha_{1}^{2}+\alpha_{2}^{2}\right) P^{(3 / 4)}} Q y K_{1}\left\{\begin{array}{c}
2 Q y\left[3 \alpha_{2}^{2}\left(32 a^{2}-40 a x+35 x^{2}\right)+\alpha_{1}^{2}\left(96 a^{2}-120 a x+105 x^{2}-70 \alpha_{2}^{2} y^{2}\right)\right] \cos \left((3 / 2) \tan { }^{-1} M\right)+ \\
\sqrt{2} \alpha_{1}^{2}\left[128 a^{3}-96 a^{2} x+60 a^{2} x-30 \alpha_{2}^{2} y^{2}(4 a-7 x)-35 x^{3}\right] \sin \left((3 / 2) \tan ^{-1} M\right)+ \\
\sqrt{2} \alpha_{2}^{2}\left(128 a^{3}-96 a^{2} x+60 a x^{2}-35 x^{3}\right) \sin \left((3 / 2) \tan ^{-1} M\right)
\end{array}\right\},
\end{aligned}
$$

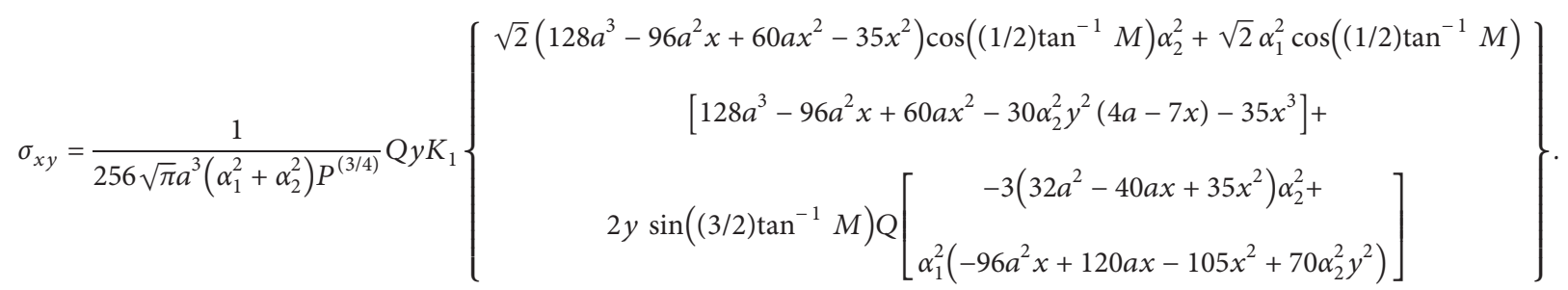

\section{Data Availability}

The data used to support the findings of this study are available from the corresponding author upon request.

\section{Conflicts of Interest}

The authors declare that there are no conflicts of interest.

\section{Acknowledgments}

The study was supported by the National Natural Science Foundation of China (NSFC grant nos. 51774018 and 51478027), the “973” Key State Research Program (grant no. 2015CB0578005), and Program for Changjiang Scholars and Innovative Research Team in University (grant no. IRT17R06).

\section{References}

[1] G. I. Barenblatt, "The mathematical theory of equilibrium cracks in brittle fracture," Advances in Applied Mechanics, vol. 7, pp. 55-129, 1962.

[2] A. Hillerborg, M. Modéer, and P.-E. Petersson, "Analysis of crack formation and crack growth in concrete by means of fracture mechanics and finite elements," Cement and Concrete Research, vol. 6, no. 6, pp. 773-781, 1976.

[3] Y. S. Jenq and S. P. Shah, "Two parameter fracture model for concrete," Journal of Engineering Mechanics, vol. 111, no. 10, pp. 1227-1241, 1985.

[4] K. B. Broberg, "Crack-growth criteria and non-linear fracture mechanics," Journal of the Mechanics and Physics of Solids, vol. 19, no. 6, pp. 407-418, 1971.

[5] K. B. Broberg, "The cell model of materials," Computational Mechanics, vol. 19, pp. 447-452, 1996.

[6] K. B. Broberg, Cracks and Fracture, Academic Press, London, UK, 1999.

[7] K. B. Broberg, "A smooth dissipative region model," International Journal of Fracture, vol. 113, no. 3, pp. 3-8, 2002.

[8] K. B. Broberg, "On the behaviour of the process region at a fast running crack tip," in High Velocity Deformation of Solids, K. Kawata and J. Shioiri, Eds., Springer-Verlag, Berlin, Heidelberg, pp. 182-194, 1979.

[9] J. Fineberg, S. P. Gross, M. Marder, and H. L. Swinney, "Instability in dynamic fracture," Physical Review Letters, vol. 67 , no. 4, pp. 457-460, 1991.

[10] J. R. M. Radok, "On the solution of problems of dynamic plane elasticity," Quarterly of Applied Mathematics, vol. 14, no. 3, pp. 289-298, 1956.

[11] T. Y. Fan, Defect and Fracture Theory of Solid and Soft Materials (In Chinese), Science Press, Beijing, China, 2014. 
[12] P. C. Paris, "Secondary elastic crack tip stresses which may influence very slow fatigue crack growth," Fatigue \& Fracture of Engineering Materials \& Structures, vol. 25, no. 8-9, pp. 791-794, 2002.

[13] H. Tada and P. Paris, "Secondary elastic crack tip stresses which may influence very slow fatigue crack growth-additional results," International Journal of Fatigue, vol. 27, no. 10-12, pp. 1307-1313, 2005.

[14] V. Z. Parton and V. G. Boriskovsky, Dynamic Fracture Mechanics, Vol. 2, Hemisphere Publishing Corporation, London, UK, 1989.

[15] G. M. Seed and D. Nowell, "Use of the distributed dislocations method to determine the T-stress," Fatigue \& Fracture of Engineering Materials and Structures, vol. 17, no. 5, pp. 605-618, 1994. 